\title{
Cross-linking at the Slit Lamp-Why Moving Corneal Cross-linking from the Operating Room to an Office-based Procedure Makes a Difference
}

Farhad Hafezi

ELZA Institute, Zurich, Switzerland

DOI: https://doi.org/10.17925/USOR.2020.13.2.55

$\mathrm{C}$ orneal cross-linking (CXL) is a procedure that combines stromal riboflavin saturation and ultraviolet (UV) light to strengthen the cornea. CXL is typically performed in an operating room. The UV-riboflavin photochemical reaction also kills pathogens, and CXL is even used to treat infectious keratitis, raising the question: do you need to perform CXL in a sterile operating room? There is a trend to move small surgical procedures out of the operating room and into minor procedure rooms or the doctor's office, and CXL is an obvious candidate for this. The benefits of avoiding the operating room in terms of cost and resource use are manifest. However, traditional CXL involves the patient lying supine for 30 minutes, while UV irradiation is applied. For CXL to be performed at, for example, the near-ubiquitous slit lamp in an office setting, several hurdles needed to be cleared, including reducing the time a patient needs to sit at the slit lamp, and understanding whether or not riboflavin settles under gravity when a patient is sat upright during CXL. This article reviews those potential obstacles and how they have been overcome, and the benefits of bringing CXL technology to clinics that do not have easy access to operating rooms for the treatment of corneal ectasia and infectious keratitis, in both developing and developed countries.

\section{Keywords}

Corneal cross-linking, slit lamp, corneal ectasia, infectious keratitis, keratoconus

Disclosure: Farhad Hafezi is the co-founder of the Light for sight foundation, which administrates the K-MAP study, and is Chief Scientific and Medical Officer of EMAGine AG, which markets a slit lamp-mountable cross-linking device.

Review Process: Double-blind peer review.

Compliance with Ethics: This article is an opinion piece and does not report on new clinical data, or any studies with human or animal subjects performed by the author.

Authorship: The named author meets the International Committee of Medical Journal Editors (ICMJE) criteria for authorship of this manuscript, take responsibility for the integrity of the work as a whole, and has given final approval for the version to be published.

Access: This article is freely accessible at touchOPHTHALMOLOGY.com (c) Touch Medical Media 2020

Received: November 9, 2020

Accepted: November 19, 2020

Published Online: December 18, 2020

Citation: US Opthalmic Review. 2020;13(2):55-7

Corresponding Author: Farhad Hafezi,

ELZA Institute AG, Webereistrasse 2, 8953 Dietikon,

Zurich, Switzerland. E: fhafezi@elza-institute.com

Support: No funding was received in

the publication of this article.
Most surgical procedures are performed in the operating room, for obvious reasons. Operating rooms are sterile, they should be stocked with all of the equipment and materials you might need, and the operating-room staff are trained and ready to assist as and when they are needed. But for the sake of convenience, many small surgeries are increasingly being performed in smaller, office-based procedure rooms rather than an operating room. This is a growing trend across medicine, and ophthalmology is no different. For example, it has been shown that office-based cataract surgery can be as safe as cataract surgery performed in the operating room of an ambulatory surgical center, with no differences in adverse events, endophthalmitis rates, or visual outcomes. ${ }^{1}$ Moving from an operating room to an office-based procedure, or even the treating doctor's office, comes with another benefit: lower costs. If you do not need the infrastructure that is present in an operating room to do your procedure, and you can address the concerns about working in a clean, but not sterile environment, then the cost savings can be considerable.

Moving away from and operating room and lowering costs brings about another benefit: broadening access to that treatment. Low-to-middle income countries (LMICS) have populations that are mostly rural, with low incomes and highly restricted access to healthcare interventions. Operating rooms are present in hospitals; in LMICS, hospitals are located in the major cities and the procedures are usually too expensive for many to afford. Lowering these barriers to care can result in a great deal more people who need care are actually receiving it.

\section{Moving corneal cross-linking out of the operating room}

$\mathrm{CXL}$ is a procedure that has historically been performed inside operating rooms. ${ }^{2}$ However, our research group and collaborators have been working for over half a decade to deliver a technology that allows us to perform CXL at the near-ubiquitous slit lamp found in the ophthalmologist's office, rather than in the operating room. When we perform CXL at the slit lamp, we are performing the same procedure as CXL in the operating room, using the same ultraviolet (UV) irradiation settings, the same concentration of riboflavin, and producing the same effect (Figure 1). The photochemical reaction between the UV energy, riboflavin, oxygen, and the molecules in the stroma does not care whether a person is sitting at a slit lamp or lying in a reclining chair in an operating room.

However, there are still concerns regarding moving CXL procedures to the office. The most apparent is that the surgery will not be performed in a sterile environment, which could result in infection. 
A

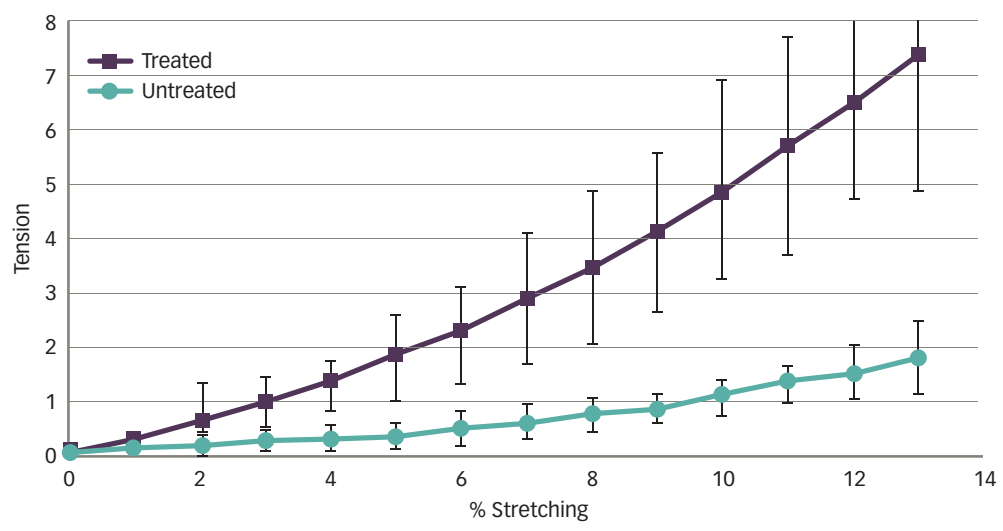

Biomechanical stiffening

C

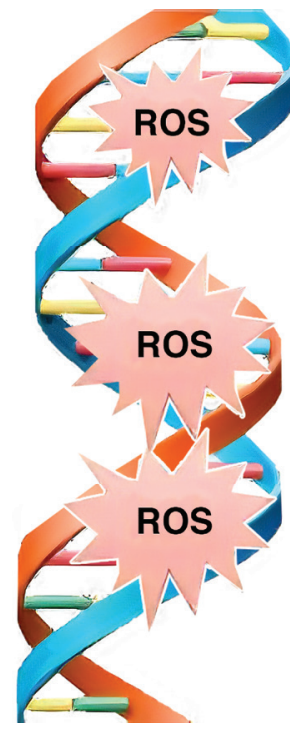

DNA/RNA intercalation

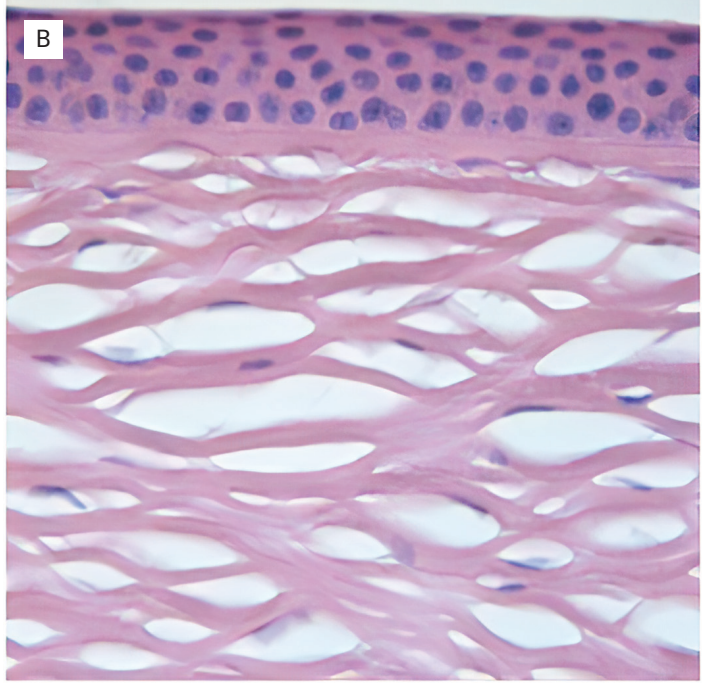

Steric hindrance

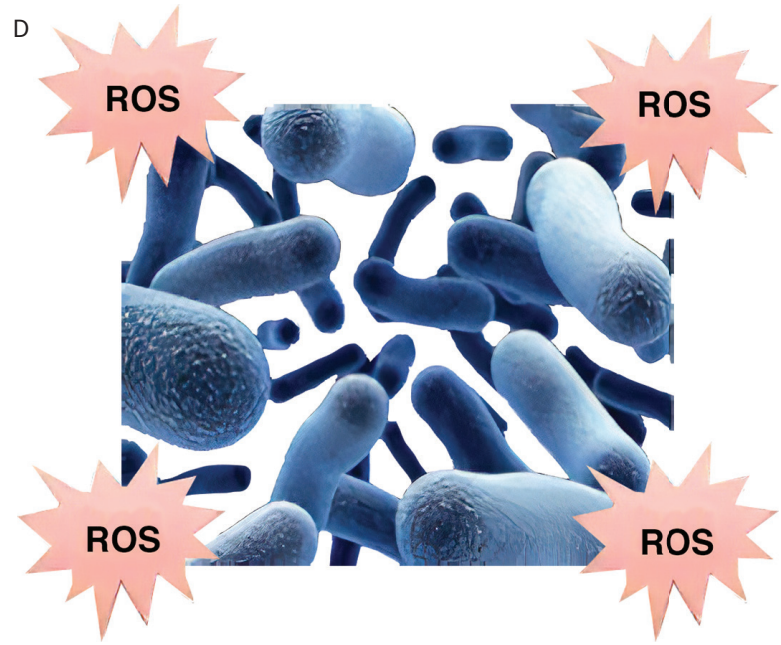

Oxidative stress

A: Biomechanical stiffening (through the covalent cross-linking of stromal molecules together); B: Steric hindrance (fewer sites where proteases can bind are available once the stromal molecules are cross-linked); C: Nucleic acid intercalation (inhibiting pathogen replication); D: Oxidative stress (damaging pathogen cell membranes).

ROS = reactive oxygen species.

Furthermore, The length of time required for the patient to sit at the slit lamp might prove too long; the classic CXL (Dresden) protocol involves 30 minutes of irradiation. ${ }^{3}$ Thirdly, gravity might start to affect the distribution of riboflavin in the eye, resulting in uneven cross-linking. These concerns are addressed below.

\section{The sterilizing effects of corneal cross-linking}

When riboflavin absorbs the energy from a UV photon, it generates reactive oxygen species (ROS); short-lived chemical species that react rapidly with anything nearby. ${ }^{4}$ The therapeutic effect in $\mathrm{CXL}$ for corneal ectasias, like keratoconus, is that ROS covalently bind molecules together within the corneal stroma (mostly collagen), ${ }^{2}$ which makes the cornea stiffer and stronger, thereby slowing or stopping the progression of the ectasia.
However, ROS also damage the cell membranes and nucleic acids of pathogens. In addition to this, UV light has a sterilizing effect of its own. ${ }^{5}$ When you perform CXL to treat ectasia, both phenomena occur at the same time during the procedure. In essence, CXL sterilizes the cornea. ${ }^{6} \mathrm{CXL}$ is so effective at this that it is now being used to treat infectious keratitis in a process known as "photoactivated chromophore for keratitis-CXL" (PACK-CXL).

Since CXL makes the cornea sterile and antibiotic prophylaxis is given immediately upon completion. CXL that is performed at a slit lamp in a doctor's office should be as safe as CXL performed in a sterile operating room, and should not result in an increase in rates of postoperative infectious complications. A current study is ongoing to investigate this 
Those who are still concerned about the risk of infection may take a clean (but not necessarily sterile) approach to the environment around the slit lamp where the CXL will be performed, in the same way that retinal specialists apply intravitreal injections.

\section{Procedural time required for corneal cross-linking}

In our experience, patients are quite happy to sit at the slit lamp and undergo $\mathrm{CXL}$ for as long as is necessary to receive their treatment. We take the time to ensure that the slit lamp, table, and chair are all adjusted correctly to make the patient as comfortable as possible during the procedure. Additionally, in almost all cases, CXL for 30 minutes at a UV-A energy intensity of $3 \mathrm{~mW} / \mathrm{cm}^{2}$ is not necessary. By increasing the intensity of illumination, you can directly and proportionately reduce the time you need to irradiate the eye. Ten minutes at $9 \mathrm{~mW} / \mathrm{cm}^{2}$ crosslinks the patient's cornea effectively enough to treat an ectasia, ${ }^{8}$ and this period is well within most people's comfort zone. Studies show that the treatment of infectious keratitis can be effective with 3 minutes of UV irradiation. 9.10

\section{The distribution of riboflavin in the eye}

An experiment performed on corneas in horizontal and vertical positions found that there was no sedimentation or significant alteration in riboflavin concentration in the corneal stroma of those sat in the vertical position, even after one hour; twice the duration of the Dresden protocol. ${ }^{11}$

\section{The global impact of PACK-CXL}

The availability of a battery-operated, portable, slit lamp-mountable cross-linking device (C-Eye; EMAGine AG, Zug, Switzerland; not available in the USA), which is approved for use in Europe, will undoubtably make CXL for ectasia more cost effective and more accessible for tertiary care centers. This combination of lower costs and care in the community setting will democratize access to CXL. Despite keratoconus being far more prevalent than previously thought, the other application of cross-linking, PACK-CXL, has a far greater potential to make an impact on the world.

Infectious keratitis, which the World Health Organization has called a "silent epidemic," ${ }^{12,13}$ is one of the leading global causes of visual impairment, especially in LMICS. It can progress rapidly, and immediate detection and treatment are crucial. Most infectious keratitis cases in temperate European countries are bacterial in origin, but in LMICs (particularly those with humid climates), many of these cases are of fungal etiology, or worse, fixed infections (i.e. bacterial and fungal). ${ }^{14}$ This is compounded by increasing antimicrobial resistance, reducing our armamentarium of effective drugs. Treating infectious keratitis with inappropriate or ineffective medication (e.g. an antibiotic for fungal infection), in addition to "wasting" precious time, can potentially increase the risk of corneal sequelae. Instead of a broad-spectrum antibiotic, broad-spectrum antimicrobials-preferably those to which microorganisms are not resistant-would be required.

As PACK-CXL is pathogen-agnostic, it could be viewed as a broadspectrum antimicrobial therapy, effective against both bacteria (including methicillin-resistant Staphylococcus aureus and Pseudomonas) and fungi. Finally, one of the positive side effects of CXL is that it increases corneal resistance to digestion by pathogens and potentially reduces the size of an eventual scar. ${ }^{15}$

\section{Evidence}

PACK-CXL has recently been evaluated in a randomized, controlled, multicenter, phase III, interventional prospective clinical trial (Hafezi et al., NCT02717871; in preparation), where patients were randomized to receive either standard-of-care treatment with antimicrobials, or PACK-CXL alone. If the patients who received PACK-CXL worsened after 1 day, they then received antimicrobials, and PACK-CXL was considered a treatment failure. Initial results indicate that PACK-CXL is as effective as antimicrobial therapy at treating infectious keratitis of bacterial or fungal origin, although the time to complete re-epithelialization was longer. Nevertheless, this research showed that PACK-CXL was able to cure infectious keratitis, without antibiotics, after a single treatment.

There is no suggestion that PACK-CXL should be used as monotherapy in routine clinical practice when there are effective antimicrobial agents available. However, in developed countries, it is an interesting option as it can reduce scar size and work in antimicrobial-resistant infections. The greatest value of PACK-CXL will be seen in LMICS, where it is difficult (or even impossible) to obtain antibiotics and access a doctor with an operating room and a cross-linking device. PACK-CXL at the slit lamp brings a treatment that is ordinarily performed in an operating room, in a hospital, in a major urban location, to anywhere an ophthalmologist and a slit lamp can travel.

\section{Conclusion}

CXL at the slit lamp has many advantages: it reduces costs for doctors and patients alike, and it brings much-needed treatment to a larger population. PACK-CXL at the slit lamp goes even further in terms of the number of people it can help, potentially saving the sight of thousands of people who would have previously gone blind due to infectious keratitis. In an ideal world, people should not have to go blind because of their location or income, when a treatment is available. $\square$
1. lanchulev T, Litoff D, Ellinger D, et al. Office-based cataract surgery: population health outcomes study of more than 21,000 cases in the United States. Ophthalmology. 2016;123:723-8.

2. Randleman JB, Khandelwal SS, Hafezi F. Corneal cross-linking. surv Ophthalmol. 2015;60:509-23.

3. Wollensak G, Spoerl E, Seiler T. Riboflavin/ultraviolet-a-induced collagen crosslinking for the treatment of keratoconus. Am J Ophthalmol. 2003;135:620-7.

4. McCall AS, Kraft S, Edelhauser HF, et al. Mechanisms of corneal tissue cross-linking in response to treatment with topical riboflavin and long wavelength ultraviolet radiation (UVA). Invest Ophthalmol Vis Sci. 2010:51:129-38.

5. Kumar V, Lockerbie O, Keil SD, et al. Riboflavin and UV-light based pathogen reduction: extent and consequence of DNA damage at the molecular level. Photochem Photobiol. 2004;80:15-21.
6. Ehlers N, Hjortdal J, Nielsen K, Søndergaard A. Riboflavin-UVA treatment in the management of edema and nonhealing ulcers of the cornea. J Refract Surg. 2009;25:S803-06.

7. Hafezi F, Randleman JB. PACK-CXL: Defining CXL for infectious keratitis. J Refract Surg. 2014;30:438-9.

8. Lang PZ, Hafezi NL, Khandelwal SS, et al. Comparative functional outcomes after corneal crosslinking using standard, accelerated, and accelerated with higher total fluence protocols. Cornea. 2019;38:433-41.

9. Richoz O, Kling S, Hoogewoud F, et al. Antibacterial efficacy of accelerated photoactivated chromophore for keratitiscorneal collagen cross-linking (PACK-CXL).J Refract Surg. 2014:30:850-4

10. Knyazer B, Krakauer Y, Baumfeld Y, et al. Accelerated corneal cross-linking with photoactivated chromophore for moderate therapy-resistant infectious keratitis. Cornea. 2018;37:528-31. 11. Salmon B, Richoz O, Tabibian D, et al. CXL at the slit lamp: no clinically relevant changes in corneal riboflavin distribution during upright UV irradiation. J Refract Surg. 2017;33:281.

12. Whitcher JP, Srinivasan M. Corneal ulceration in the developing world-a silent epidemic. Br J Ophthalmol. 1997;81:622-3.

13. World Health Organization. Antimicrobial resistance: global report on surveillance 2014. Available at: www.who.int/drugresistance/ documents/surveillancereport/en/ (accessed 19 November 2020).

14. Bongomin F, Gago S, Oladele RO, Denning DW. Global and multi-national prevalence of fungal diseases_estimate precision. multi-national pre

15. Hammer A, Richoz O, Mosquera S, et al. Corneal biomechanical properties at different corneal collagen cross-linking (CXL) Irradiances. Invest Ophthalmol Vis Sci. 2014;55:2881-4. 